\title{
Nursing Mode for Advanced Gastric Carcinoma with Combined Application of Domestic Tegafur Gimeracil Oteracil Potassium and Oxaliplatin
}

\author{
X. BA, J. HAN*, G. ZHAO ${ }^{1}$ AND YU MU ${ }^{1}$ \\ Department of Radiotherapy, ${ }^{1}$ Department of Colorectal and Anal Surgery, The Second Hospital of Jilin University, Changchun \\ 130041, China
}

Ba et al.: Nursing Method for Advanced Gastric Carcinoma with Combined Applications

\begin{abstract}
To observe and analyze the nursing method for advanced gastric carcinoma with combined application of domestic tegafur gimeracil oteracil potassium and oxaliplatin. 160 patients who had been diagnosed with advanced gastric carcinoma in our hospital were selected as research objects and all of them were applied with therapy of domestic tegafur gimeracil oteracil potassium and oxaliplatin. During treatment, targeted nursing mode was implemented to overcome relevant toxic reaction. The nursing effect was observed and analyzed. After implementation of nursing, all patients were applied with chemotherapy regimen successfully and the total treatment effective rate reached $78.13 \%$. During treatment with combined application of domestic tegafur gimeracil oteracil potassium and oxaliplatin, the main toxic reactions were peripheral neurotoxicity, leukopenia, thrombocytopenia, diarrhea, nausea, vomiting, phlebitis, etc. The comparison of mental status score before and after nursing showed that the mental status was significantly better after nursing, $p<0.05$. During treatment of advanced gastric carcinoma with combined application of domestic tegafur gimeracil oteracil potassium and oxaliplatin, implementing targeted nursing can significantly reduce toxic reactions.
\end{abstract}

Key words: Domestic tegafur gimeracil oteracil potassium, oxaliplatin, advanced gastric carcinoma, nursing model

Gastric carcinoma is a type of malignant tumor on gastric mucosal epithelium, which tops the incident rate of all types of malignant tumor. According to relevant statistics, there is a significant regional disparity on incident rate of gastric carcinoma in China. Compared with southern area of China, the northwest and eastern coastal areas suffer higher incident rate of gastric carcinoma ${ }^{[1-3]}$. Gastric carcinoma normally occurs to people over $50 \mathrm{y}$ old and to males more than females. With the change of living mode, diet structure and the increase of work stress, patients with gastric carcinoma become younger now a day's ${ }^{[4]}$. Gastric carcinoma (fig. 1) can occur to any part of gastric area, but gastric antrum (also known as pylorus, fig. 2) is the site where gastric carcinoma occurs most frequently. Gastric carcinoma occurring on pylorus can also affect gastric curvature, gastric curvature and anterior and posterior walls. Many gastric carcinomas are belonging to adenocarcinoma. In the early stage of gastric carcinoma, there are only some symptoms (belching, upper abdominal discomfort) that are similar to the symptoms of gastritis, stomach ulcer and other chronic gastric diseases, therefore, the gastric carcinoma is often misdiagnosed or neglected. Advanced gastric carcinoma means the carcinoma tissues have infiltrated into submucosa, entering into muscular layer or penetrating muscular layer into serous membrane. In this stage, the gastric carcinoma can be divided into polyp type, ulcer

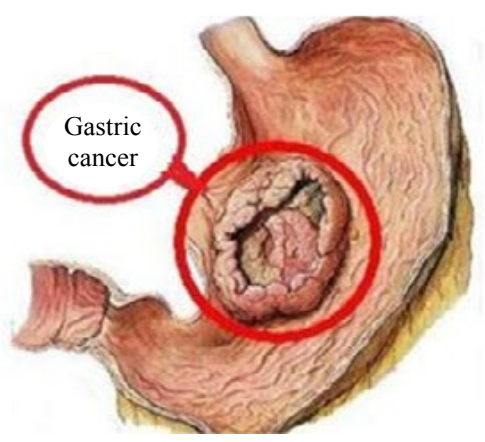

Fig. 1: Gastric carcinoma 
type and infiltration type by macroscopic observation. Infiltrative gastric carcinoma is often accompanied with thickening and hardening of gastric wall and formation of linitis plastica. The main approach to treat advanced gastric carcinoma is chemotherapy, which can improve life quality and prolong survival time of patients in the largest degree. Currently, the therapy based on 5 fluorouracil (5-Fu) and first type of drugs is commonly applied as first line treatment. This study observe the nursing effect of advanced gastric carcinoma with combined application of domestic tegafur gimeracil oteracil potassium and oxaliplatin, which provide valuable reference for treatment and nursing of advanced gastric carcinoma. 160 patients who had been confirmed and treated for advanced gastric carcinoma in our hospital from January 2014 to February 2018 were selected as research objects. The inclusion criteria are: Stage IIIB-IV period of gastric carcinoma (fig. 3) by pathological examination or imageological examination; Karnofsky score is 60 points or above; expected survival is 3 mo or more; normal results of electrocardiogram (ECG) and blood routine examination; normal hepatic and renal function. Exclusion criteria are: patients with contraindication to chemotherapy, the mentally disordered, patients with insufficient liver, heart and kidney function. Among all selected patients, there were 104 males and 56 females, with age range from $32-70 \mathrm{y}$ old, averaging at 58.3 \pm 8.2 . All selected patients enjoyed the right to know and signed the informed consent. The patients with advanced gastric carcinoma were treated with combined application of domestic tegafur gimeracil oteracil potassium and oxaliplatin. The intravenous injection of oxaliplatin $\left(130 \mathrm{mg} / \mathrm{m}^{2}\right)$ was performed in d 1; Domestic tegafur gimeracil oteracil potassium $\left(40 \mathrm{mg} / \mathrm{m}^{2}\right)$ was applied in twice, d 1-d 14. A complete course of treatment contains $21 \mathrm{~d}$, all patients were subjected to chemotherapy for three cycles. First, mental nursing was applied. Patients with advanced gastric carcinoma normally have been applied with multiple chemotherapies. Due to insufficient therapeutic response and disease pain, the patients will show various negative emotions, such as anxiety, nervousness, insecurity, panic, depression, etc. If timely measures are not taken to remove these negative emotions, the treatment will be affected in certain degree. Nursing staff should communicate more with patients to know the mental status and conduct targeted guidance. For example, talking, walking and listening to music are effective approach to distract attention. Moreover, speaking more about successful treatment cases can encourage patients to establish positive, optimistic and courageous belief to overcome disease and improve compliance to treatment ${ }^{[5,6]}$. Second, strengthening nursing for peripheral neurotoxicity. Symptoms of peripheral neurotoxicity mainly include dysesthesia and paresthesia. Nursing staff should instruct patients and relatives to take on face mask and gloves to keep warm within at least $1 \mathrm{w}$ after taking the medication. They are forbidden to touch iron products or aluminum products. If necessary, dexamethasone and B vitamins should be applied. Next, strengthening nursing for myelosuppression. During the period of chemotherapy, indoor should be maintained clean and fresh and the indoor environment should be maintained clean and tidy. Open window for ventilation and change the bed sheet every day. The blood routine of patients should be monitored periodically. All operations should be conducted according to aseptic standard. If the leucocyte level of patient is $3 \times 109 / \mathrm{L}$, the patient is required to take bed rest, with less frequency time of visits. Ultraviolet radiation disinfection should be conducted

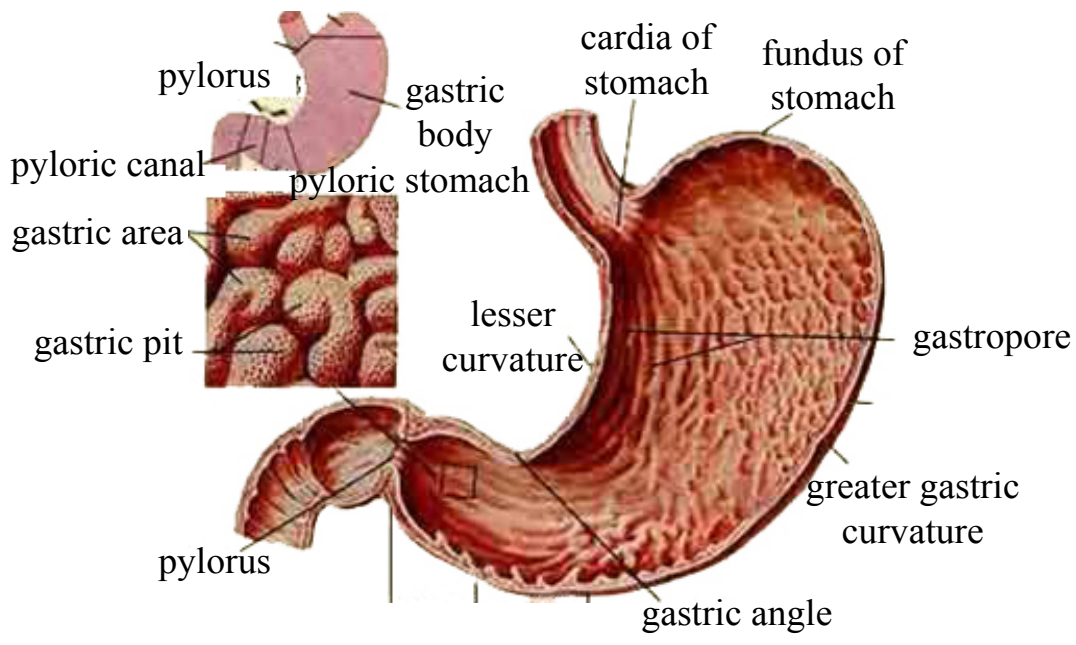

Fig. 2: Gastric antrum 
twice a day for ward, meanwhile treatment should be carried out to promote leucocytes. If the leucocyte level is under $50 \times 109 / \mathrm{L}$, the patient is required to take less exercises to prevent collision, meanwhile strict monitoring should be conducted to check if there is bleeding ${ }^{[7]}$. Last, nursing for gastrointestinal reaction was done. During half an hour before chemotherapy, intravenous injection of granisetron (3 $\mathrm{mg})$ or ondansetron $(8 \mathrm{mg})$ should be conducted according to doctor's orders to prevent gastrointestinal reaction. If vomiting occurs, the color, amount, character of vomitus need to be observed, meanwhile dehydration level should be evaluated. If necessary, electrolyte checking and fluid replacement therapy are conducted ${ }^{[8]}$. In addition, strengthening nursing for phlebitis. In the process of transfusion, the vein with larger outside diameter and better elasticity is selected and dorsal joint should be avoided as much as possible. Alternatively, deep vein catheterization can be used to prevent mechanical phlebitis or tissue necrosis and thus secure blood vessel safety. During the period of common venipuncture, it is needed to implement intravenous injection in a planned way and prevent multiple injections on the same vein. After finishing injection operation, a regular frequent visiting should be done to check if there is drug extravasation. If drug extravasation is observed, the infusion of drug should be terminated immediately and the drug residue should be drawn back by connecting with a $5 \mathrm{ml}$ injection syringe. The total treatment effective rate of patients

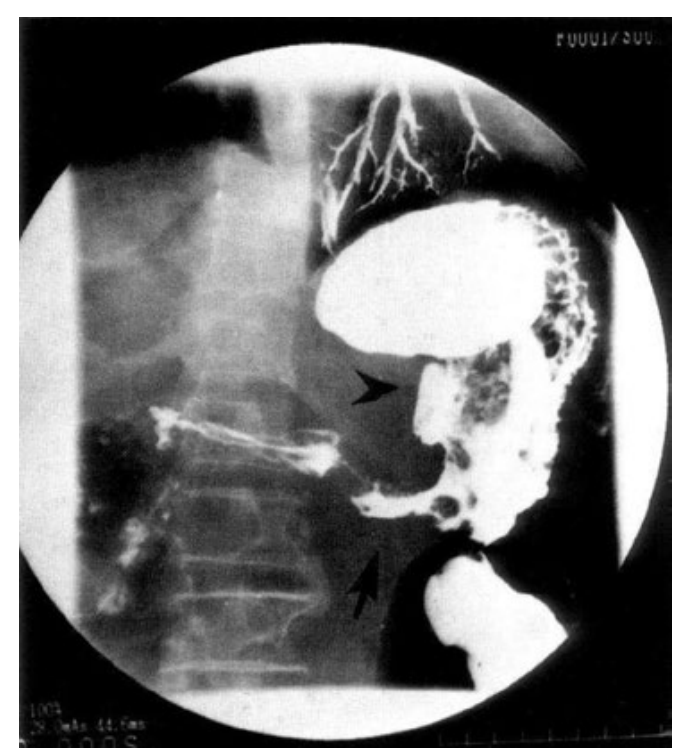

Fig. 3: Imageological examination was recorded and evaluated according to World Health Organization (WHO) criteria including complete remission, partial remission, stable disease and disease progression. Neurotoxic reactions were evaluated according to Extremely Vulnerable Individual (EVIF) criteria. Patient's mental status was evaluated using Zung self-rating anxiety scale (SAS) and Zung selfrating depression scale (SDS). SPSS 21.0 software was used for statistical analysis. The measurement data was expressed in the form of mean \pm average $\left(\overline{\mathrm{x}}^{ \pm} \mathrm{s}\right)$ and intergroup difference was compared by $t$ test. The enumeration data was expressed by natural number (n) and percentage $(\%)$ and the intergroup difference was tested by $\mathrm{x}^{2}$. The difference was of statistical significance when $\mathrm{p}<0.05$. As shown in Table 1 , after combined application of chemotherapy and scientific nursing care, the total treatment effective rate reaches $78.13 \%$. Table 2 shows the rate of toxic and adverse reactions in treatment of advanced gastric carcinoma with combined application of domestic tegafur gimeracil oteracil potassium and oxaliplatin. As shown in Table 3, the SAS and SDS scores after nursing are better than that before nursing, $p<0.05$. China is a country with high incident rate of gastric carcinoma. Gastric carcinoma is a malignant tumor causing top death rate. With the progression of gastric carcinoma, the tumor tissue may penetrate into stomach muscle layer, serosal layer or extra membrane, then we can call it as advanced gastric carcinoma despite of tumor size or metastasis or not. Research indicates that Helicobacter pylori (fig. 4) infection is closely correlated with gastric carcinoma. For patients with advanced gastric carcinoma, chemotherapy is the major treatment approach. The combined application of domestic tegafur gimeracil oteracil potassium and oxaliplatin has been widely used in treatment of advanced gastric carcinoma and significant therapeutic effect has been achieved. During the period of chemotherapy ${ }^{[9-12]}$, a scientific nursing model should be implemented to guarantee smooth implementation of chemotherapy and reduce compliances. In this study, a comprehensive nursing mode was given to all selected patients, involving mental nursing, environmental nursing, nursing for peripheral neurotoxicity, myelosuppression and phlebitis, etc. Through implementing systematic, scientific and standard nursing services, the toxic and adverse effects of chemotherapy drugs can be reduced,

TABLE 1: TOTAL TREATMENT EFFECTIVE RATE [n (\%)]

\begin{tabular}{lcccccc}
\hline Case number & $\begin{array}{c}\text { Complete } \\
\text { remission }\end{array}$ & Partial remission & Stable disease & $\begin{array}{c}\text { Disease } \\
\text { progression }\end{array}$ & $\begin{array}{c}\text { Total treatment } \\
\text { effective rate }\end{array}$ \\
\hline 160 & 40 & 85 & 20 & 15 & $125(78.13)$ \\
\hline
\end{tabular}


TABLE 2: TOXIC AND ADVERSE REACTIONS IN TREATMENT OF ADVANCED GASTRIC CARCINOMA WITH COMBINED APPLICATION OF DOMESTIC TEGAFUR GIMERACIL OTERACIL POTASSIUM AND OXALIPLATIN [n (\%)]

\begin{tabular}{|c|c|c|c|}
\hline \multirow{2}{*}{ Toxic and adverse reactions } & \multicolumn{2}{|c|}{ Toxicity level } & \multirow{2}{*}{ Rate (\%) } \\
\hline & Level 0-I & Level II-IV & \\
\hline Peripheral neurotoxicity & 56 & 26 & $82(51.25)$ \\
\hline Leukopenia & 44 & 12 & $56(35.00)$ \\
\hline Thrombocytopenia & 36 & 4 & $40(25.00)$ \\
\hline Nausea and vomiting & 86 & 9 & $95(59.38)$ \\
\hline Oral mucositis & 34 & 8 & $42(26.25)$ \\
\hline Phlebitis & 16 & 2 & $18(11.25)$ \\
\hline Diarrhea & 17 & 2 & $19(11.88)$ \\
\hline Erythrasma & 14 & 1 & $15(9.38)$ \\
\hline
\end{tabular}

TABLE 3: COMPARISON OF SAS AND SDS SCORES ( $\overline{\mathbf{x}} \pm \mathbf{s})$

\begin{tabular}{lccc}
\hline Time & Case number & SAS score & SDS score \\
\hline After nursing & 160 & $41.70 \pm 4.25$ & $40.61 \pm 2.80$ \\
Before nursing & 160 & $48.92 \pm 5.05$ & $48.65 \pm 3.86$ \\
$\mathrm{t}$ & & 9.72 & 6.51 \\
$\mathrm{p}$ & & $<0.05$ & $<0.05$ \\
\hline
\end{tabular}

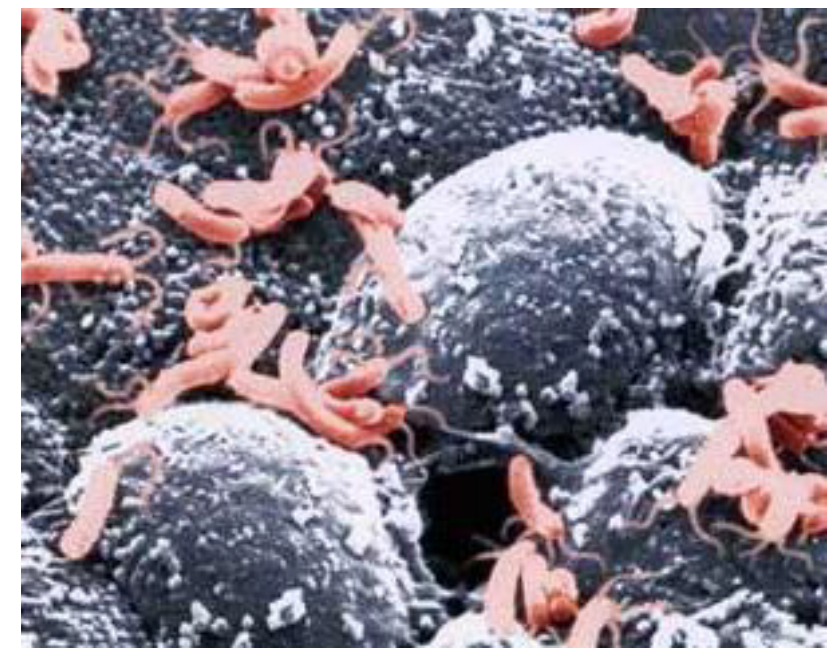

Fig. 4: Helicobacter pylori

the mental status and life quality of patients were improved. In conclusion, during treatment of advanced gastric carcinoma with combined application of domestic tegafur gimeracil oteracil potassium and oxaliplatin, implementing targeted nursing can significantly reduce toxic and adverse reactions and improved quality life of patients. Therefore, it is worth of being promoted in clinics.

\section{Conflicts of Interest:}

The authors declared no conflict of interest.

\section{REFERENCES}

1. Li X, Jin Z. Observation of short-term effects of tegafur, gimeracil and oteracil capsules combined with oxaliplatin in treatment of advanced gastric cancer. Med J Chin People Health 2014;26(4):42-4.

2. Lu J, Wang Y. Nursing care of domestic tegafur gimeracil oteracil potassium combined with oxaliplatin in the treatment of advanced gastric cancer. Chin J Mod Drug Appl 2014;8(5):183.

3. Zhang J, Xu X, Pei L. Comparison of therapeutic effect between tegafur gimeracil oteracil potassium capsule and 5-fluorouracil adjuvant therapy in treatment of advanced rectal carcinoma. Chin J Gen Surg 2015;24(9):1341-4.

4. Ushiku T, Shinozaki A, Shibahara J, Iwasaki Y, Tateishi Y, Funata N, et al. SALL4 represents fetal gut differentiation of gastric cancer, and is diagnostically useful in distinguishing hepatoid gastric carcinoma from hepatocellular carcinoma. Am J Surg Pathol 2010;34(4):533-40.

5. Chen G, Chen SM, Wang X, Ding XF, Ding J, Meng LH. Inhibition of chemokine (CXC motif) ligand 12/chemokine (CXC motif) receptor 4 axis (CXCL12/CXCR4)-mediated cell migration by targeting mammalian target of rapamycin (mTOR) pathway in human gastric carcinoma cells. J Biol Chem 2012;287(15):12132-41.

6. Liu Y. Clinical effect of combined application of tegafur gimeracil oteracil potassium capsule and oxaliplatin in treatment of advanced colorectal cancer. World Latest Med Inf 2017;17(45):135.

7. Li Y, Zhang G, Zhang M, Zhang X, Zhou X. Observation of effect of nursing intervention on improving gastroscope compliance under the guidance of health belief. Chin Gen Pract Nurs 2017;15(34):4245-9.

8. Li S, Ji M, He N, Lu Z. Application of microarray-based method for methylenetetrahydrofolate reductase (MTHFR) polymorphisms in the risk of gastric carcinoma in east China population. J Nanosci Nanotechnol 2007;7(9):3245-9.

9. Hild C, Talha-Vautravers A, Hoefler P, Zirabe S, Bellocq JP, Mathelin C. Metastatic breast cancer to the stomach: an uncommon evolution of breast carcinoma. Gynecol Obstet Fertil 2013;42(1):47-50.

10. Liu S, Sun X, Xu X, Lin F. Comparison of endocrine therapies in hormone receptor-positive and human epidermal growth factor receptor 2-negative locally advanced or metastatic breast cancer: A network meta-analysis. J Breast Cancer 
2020;23(5):460-83.

11. Liu J, Wang C, Cao L, Li S. Treatment of advanced intrahepatic cholangiocarcinoma with sintilimab combined with tegafurgimeracil-oteracil potassium capsules (S-1): a case report. Ann Palliat Med 2020;9(2):497-503.

12. Tan YE, Xing Y, Ran BL, Zhang C, Pan SW, An W, et al. LINC01235-TWIST2 feedback loop facilitates epithelial mesenchymal transition in gastric cancer by inhibiting THBS2. Aging 2020;12(24):25060-75.
This is an open access article distributed under the terms of the Creative Commons Attribution-NonCommercial-ShareAlike 3.0 License, which allows others to remix, tweak, and build upon the work non-commercially, as long as the author is credited and the new creations are licensed under the identical terms

This article was originally published in a special issue,

"Evolutionary Strategies in Biomedical Research and

Pharmaceutical Sciences" Indian J Pharm Sci 2021:83(3)

Spl issue;206-210 